\title{
DISCUSSION.
}

\section{RECENT DEVELOPMENTS IN THEORY OF EMOTION.}

Considerable interest attaches to the recent artıcles on Emotion by Professors Baldwin and Dewey. Both these writers are in favor of the James theory, but each has arrived at his results in his own way, and has his own view with regard to the weak points of the original statement of the theory and the value of the emendations that have recently been made. I do not propose, however, to deal with all the points of interest here involved. I shall merely discuss briefly the main arguments brought forward.

Professor Dewey contends that "all so-called expressions of emotion are in reality the reduction of movements and stimulations originally useful into attitudes" (Psy. Rev., I, 6, 569). They are explicable by reference to useful acts, and therefore cannot be deduced from an antecedent emotion. Now, the term 'expression of emotion ' is ambiguous, not only in denotation, but also in connotation. Few would assert that the bodily changes usually signified by this mode of speech are primarily intended to show that a certain individual has a particular emotion. The majority of psychologists would agree that the movements in question are in the main teleologically conditioned. The question is, whether you can conclude from this that they cannot be regarded as the outcome of some antecedent emotion. Professor Dewey's argument depends on the assumption that no useful action can be explained by reference to emotion. But surely the natural supposition is that so prominent a fact of consciousness has some function, and therefore directly or indirectly influences our actions. Professor Dewey himself says that "hope, fear, delight, sorrow, terror, love are too important and too relevant in our lives to be in the main the 'feel' of bodily attitudes which have themselves no meaning" (PSY. REv., I, $6,5^{6} 3$ ). It is hard to see how these can be important or relevant in our lives, if it is enough to dissociate an action from them to show that it has a purpose or end. I do not assert that all the bodily changes, internal and external, are the result of emotion. My contention is that it does not follow, merely because movements are 
purposive, that they have no connection with emotion; and, further, that it would be a strange thing if this conclusion were correct.

We must now consider how far the detailed account of the bodily changes supports the 'effect' theory. A distinction is drawn, implicitly at all events, between the internal organic disturbance and those outward movements which are actions in the true sense, since they are directed towards some end. With regard to the latter, no explanation is given. All that is done is to show that they are directly teleological. It is first assumed that emotion can have no connection with useful actions, and then actions of this sort are simply taken for granted. If, however, they cannot be deduced from the emotion, it is legitimate to demand some account of their origin. It cannot be too strongly emphasized that the so-called 'cause' theory has an intelligible explanation to offer, while the 'effect' theory is silent on the point. Further, as Professor Baldwin brings out so clearly, it is not easy to see how any explanation can be given, on the principles of the latter view, which will fit into a theory of development.

A complication is introduced by the fact that some of these movements, such as the crouching of fear and the clenching of the fist in anger, are reflex. It is easy to understand, however, that once these actions have been voluntarily performed under the influence of a certain emotion, they arise reflexly in circumstances similar to those with which they were first connected. The identity of crrcumstance redintegrates the old movements, and so emotion and action appear simultaneously. In these cases, of course, the bodily changes are not caused by the particular emotion which they accompany. On the other hand, these reflexes are not the only 'expressions' of emotion. The original expression of anger, for instance, was doubtless a blow. The clenching of the fist points to restraining influences. But, though we do not now use crude physical measures exclusively, civilization does not leave us altogether helpless. The covert sneer, the insulting stare, the cutting remark are at our service. These and similar purposive actions require to be accounted for. The natural thing is to-regard them as the effects of the emotion. There is an intelligible relation between them and the psychical state. An individual possessed with hate of some person will tell you that 'he feels as if he could '-do him all sort of injury. It is reasonable to suppose that this feeling conditions the action of retaliation. When it is asserted that this peculiar feeling towards the person only arrives after the action has actually taken place, we are at liberty to entertain a doubt in the 
matter. When we find that no explanation whatever is given of the appearance of the action, our doubt will scarcely be diminished.

The organic changes, then, which are directly teleological, must be regarded as falling into two classes, namely, reflexes and voluntary movements. As Professor Baldwin shows, the effect-theory does not and cannot afford any explanation of the origin of the former in the first instance. The latter can scarcely be said to be taken into account at all, for the view is not worked out in detail. Cases like fear are used as instances where the action is practically the same under all circumstances, so that the reflex and the voluntary movements coincide. ${ }^{1}$

The internal organic changes remain, and Professor Dewey really faces the question here by attempting to show how they can be accounted for without reference to an antecedent emotion. They result from "the effort of the organism to adjust its formed habits or coördinations of the past to present necessities as made known in perception or idea. The emotion is psychologically the adjustment or tension of habit and ideal, and the organic changes are the literal working out in concrete terms of the struggle of adjustment" (Psy. REv., II, I, p. $3^{\circ}$ ). The habitual reaction does not harmonize with the mode of action which the present circumstances seem to demand. Hence there is a conflict, and, as a result of this, disturbances of visceral and associated organs, "which is just what we might expect when there is a great stirring up of energy preparatory to activity, but no defined channel of discharge" (Psy. Rev., I, 6, 565). It is not easy to understand why the habitual reaction should assert itself so strongly in circumstances where it is obviously so much out of place, and insist on being 'coördinated' with the new mode of behavior. Nor is it obvious how emotion can be the 'tension of habit and ideal,' prior to action, when it is first constituted after the action has taken place (II. I. pp. 18, 22). Leaving these points undiscussed, let us ascertain the results which follow from the explanation of the internal organic agitation here given. In the first place, the course taken by the deflected energy would seem to be mechanically determined as Professor James suggests (Principles of Psycholo$g y$, II., p. 482). It will be dependent on the individual organism and its state at the moment. Professor Dewey is quick to see the effect of this. "If the bodily attitude is wholly accidental, then the emotion itself is brute and insignificant, upon a theory which

${ }^{1}$ In this connection one might safely venture the assertion that the theory under discussion would not seem so convincing if it were applied all round, instead of being stated generally, and merely illustrated by one or two favorable instances. 
holds that emotion is the "feel' of such an attitude" (I, 6, p. 563). He finds it 'more or less intolerable' that any idiopathic effects should be 'purely mechanical outpourings through the easiest available channel,' and maintains that the easiest path is determined by habits which, upon the whole, were evolved as useful (I, 6, p. 563). It would be very difficult, however, to prove that the bodily excitement could ever have been useful; and until such proof is offered, the 'intolerable' supposition of Professor James must be regarded as the more probable.

In the second place, under whatever circumstances the energy is aroused, the same amount spreading through the same organism at the same time will have the same effects. There is simply so much energy which is under a mechanical necessity to find an outlet. That the special occasion has no influence in determining the actual channels of discharge is rendered more obvious when we remember that the whole process is necessary just because action, appropriate to the particular case, has been inhibited. In every emotional state of the same intensity, therefore, the physical agitation will be practically the same; and this is a result more than serious to those who assert that the bodily changes cause the emotion. When the alleged causes are so much alike, why should the psychical effects be so widely different?

I do not criticise Professor Dewey for attempting to account for the internal organic disturbance without reference to emotion. It seems to me that he has shown that the phenomenon in question cannot be regarded as an effect of the emotion; and, further, that he has indicated the right principle ${ }^{1}$ by which its origin is to be understood. My point is that the consequences have been shown to be disastrous to the theory he has adopted. For instance, one of the great difficulties which has to be met is that the bodily changes do not vary sufficiently in the case of different emotions, while they vary too much in different instances of the same emotion. The objection on this score was formerly made on the ground of empirical observation. Now, we can not only urge that the fact is so, but give a reason why, in the nature of things, it should be as it is. The escape of deflected energy is the cause of the internal effects, and these form the greater part of the whole mass of bodily change. Since the process is under mechanical law, the effects vary with the state of the organism; and this accounts for the fact that the same

1 The principle must be worked out more fully, however, and freed from irrelevant 2dditions. In all probability, too, it will be found necessary to supplement it to some extent by others. 
emotion may at different times have different physical accompaniments. Further, under any outward circumstances a given amount of energy will always produce the same results if the inward physical conditions remain unaltered; and this explains why, in all states of equal intensity, the organic changes are sabstantially the same.

On the other hand, it seems possible for opponents of the effecttheory to state their views, so as to include and harmonize all the facts. You cannot proceed on the assumption that emotion must be either the cause or the effect of the physical changes. In the first place, the term 'physical change' is wide and vague. It covers movements, purposively reflex, voluntary, and mechanically determined. In the second place, the antithesis is false, for there is a third possibility, namely, that the psychical and physical aspects are independent of each other and yet concomitant. It is possible to hold, therefore, that some of the bodily changes are effects of the emotion, that others are independent of it, and that the emotion is in turn independent of the latter. When danger threatens, for example, it is possible to imagine that the perception is followed at once by fear and an arousal of energy, while certain actions or tendencies to action are called forth by association. All these arise simultaneously. We have at once the emotion of fear, the excitation of energy, and the tendency to crouch or run away. In the case of the first emotion of the kind, the third effect would not be present as a direct consequence of the cognition. The psychical state is a feeling with reference to the impending event prompting us to action of a certain kind with regard to it. The energy excited renders it possible for this action to be carried out with speed and vigor. If flight is out of the question, the energy diffuses itself through the organism and produces the violent organic paroxysm of intense terror. If the danger can be avoided and the means are suggested, the energy is used up for the most part in carrying out the necessary movements. Still a certain quantity always discharges itself through the body, for more energy is aroused than is absolutely necessary, and in most instances an interval must elapse before means can be found and the action put in train. Even though the purposive action is not inhibited, therefore, a certain degree of physical agitation is always present. I have purposely taken one of the cases which is most favorable to the effect-theory. The organic perturbation is not nearly so marked when, as in hate for instance, immediate and decisive action is not so vitally essential. The emotion may be as strong, however, for the physical changes vary in strength and prominence, not with the psychical concomitant, but 
with the practical demands of the situation. Further, it is only in an instance, as simple as the one I have chosen, that it can be maintained with any show of plausibility that reflex action accounts for all the movements involved. In more complicated cases more complicated actions are necessary, and it is not so easy to exclude from consideration the influence of the purely psychical aspect of the concrete emotional state.

I can only refer to Professor Baldwın's interesting paper in so far as it bears on the special point now under discussion. This writer argues that "though habit means subsiding consciousness, it is just those 'expressive' reactions which are most instinctive, that carry with them most of the vivid and disturbed consciousness we call emotion" (Psy. Rev., I, 6, 6r2). Hence he concludes that the consciousness in question cannot arise until the instinctive reactions are reported back from the periphery. This view is open to criticism on many points, but I merely wish to point out that, thanks to Professor Dewey, it can be attacked on a question of fact. The argument depends on the assumption that all 'expressive reactions' are instinctive. Some are undoubtedly, but the greater number of them are caused by the deflection of energy or the spreading of excess energy through the body. They are not instinctive at all, but simply "mechanical outpouring through the easiest drainage channels.'

We find, therefore, that nothing has been adduced on the basis of which it can be denied that emotion has a function of some kind, and causes some of the bodily movements which enter into the complete emotional state. The voluntary actions which seem to follow naturally from the peculiar psychical attitude are either left out of account altogether, or confounded with the instinctive reactions, which are themselves simply taken for granted. The explanation of the internal organic disturbance is in principle sound. It involves consequences, however, which are fatal to the effect-theory, and incidentally it destroys the special argument on the strength of which Professor Baldwin is induced to give a qualified assent to Professor James' main contention. On the other hand, opponents can accept it gratefully, and take account of it in framing their own theories.

David IRONS.

Cornell University. 